\title{
THE TRANSIT LIGHT CURVE PROJECT. V. SYSTEM PARAMETERS AND STELLAR ROTATION PERIOD OF HD 189733
}

\author{
Joshua N. Winn, ${ }^{1}$ Matthew J. Holman, ${ }^{2}$ Gregory W. Henry, ${ }^{3}$ Anna Roussanova, ${ }^{1}$ Keigo Enya, ${ }^{4}$ \\ Yuzuru YoshiI, ${ }^{5,6}$ Avi Shrorer, ${ }^{7}$ Tsevi Mazeh, ${ }^{7}$ John Asher Johnson, ${ }^{8}$ \\ Norio Narita, 9 and Yasushi Suto 9 \\ Received 2006 December 6; accepted 2006 December 28
}

\begin{abstract}
We present photometry of HD 189733 during eight transits of its close-in giant planet, and out-of-transit photometry spanning $2 \mathrm{yr}$. Using the transit photometry, we determine the stellar and planetary radii and the photometric ephemeris. Outside of transits, there are quasi-periodic flux variations with a 13.4 day period that we attribute to stellar rotation. In combination with previous results, we derive upper limits on the orbital eccentricity and on the true angle between the stellar rotation axis and planetary orbit (as opposed to the angle between the projections of those axes on the sky).

Key words: planetary systems — planetary systems: formation — stars: individual (HD 189733) — stars: rotation

Online material: machine-readable table
\end{abstract}

\section{INTRODUCTION}

For the same reason that eclipsing binary stars are important in stellar astrophysics, transiting planets play an outsized role in exoplanetary science. This can be appreciated by comparing the transiting exoplanet HD 189733b (Bouchy et al. 2005), the subject of this paper, to the arbitrarily chosen nontransiting planet HD 187123b (Butler et al. 1998). Both planets are "hot Jupiters" detected by the Doppler method. All that is known about HD $187123 \mathrm{~b}$ is its orbital period $(P=3.097$ days $)$ and minimum mass $\left(M_{p} \sin i=0.52 M_{\mathrm{Jup}}\right)$, despite $6 \mathrm{yr}$ having elapsed since its discovery. In contrast, for HD $189733 \mathrm{~b}$, discovered only $1.5 \mathrm{yr}$ ago, transit photometry has revealed the planet's radius $\left(1.15 R_{\mathrm{Jup}}\right.$; Bouchy et al. 2005; Bakos et al. 2007) and removed the $\sin i$ ambiguity in the planet's mass $\left(M_{p}=1.13 M_{\text {Jup }}\right)$. Infrared photometry during a secondary eclipse has led to a determination of the planet's $16 \mu \mathrm{m}$ brightness temperature $\left(T_{p}=1300 \mathrm{~K}\right.$; Deming et al. 2006). Most recently, spectroscopic observations during a transit have shown that the angle on the sky between the orbit normal and the stellar rotation axis is within a few degrees of zero (Winn et al. 2006).

These measurements are essential for a complete understanding of the atmospheres and interiors of hot Jupiters, as well as their formation and migration mechanisms. An even richer set of measurements can be expected in the future, from investigators pursuing transmission spectroscopy, reflected-light observations,

\footnotetext{
${ }^{1}$ Department of Physics, and Kavli Institute for Astrophysics and Space Research, Massachusetts Institute of Technology, Cambridge, MA 02139, USA.

${ }^{2}$ Harvard-Smithsonian Center for Astrophysics, Cambridge, MA 02138, USA.

3 Center of Excellence in Information Systems, Tennessee State University, Nashville, TN 37209, USA.

${ }^{4}$ Institute of Space and Astronautical Science, Japan Aerospace Exploration Agency, Sagamihara, Kanagawa 229-8510, Japan.

5 Institute of Astronomy, School of Science, University of Tokyo, Mitaka, Tokyo 181-0015, Japan.

${ }^{6}$ Research Center for the Early Universe, School of Science, University of Tokyo, Bunkyo-ku, Tokyo 113-0033, Japan.

7 Raymond and Beverly Sackler Faculty of Exact Sciences, Wise Observatory, Tel Aviv University, Tel Aviv 69978, Israel.

8 Department of Astronomy, University of California, Berkeley, CA 94720, USA.

${ }^{9}$ Department of Physics, University of Tokyo, Tokyo 113-0033, Japan.
}

and other transit-related investigations (as reviewed recently by Charbonneau et al. 2007a). One goal of the Transit Light Curve (TLC) Project is to support these efforts by refining the estimates of the planetary, stellar, and orbital parameters through highaccuracy, high-cadence photometry of exoplanetary transits. We also seek to measure or bound any variations in the transit times and light-curve shapes that would be caused by the influence of additional bodies in the system (Miralda-Escudé 2002; Agol et al. 2005; Holman \& Murray 2005). Along the way, we are exploring different techniques for photometry and parameter determination. Previous papers in this series have reported results for the exoplanets XO-1b (Holman et al. 2006), OGLE-TR-111b (Winn et al. 2007b), TrES-1 (Winn et al. 2007a), and OGLE-TR-10b (Holman et al. 2007).

This paper presents our results for HD $189733 \mathrm{~b}$, along with out-of-transit photometry spanning $2 \mathrm{yr}$. The reason for gathering out-of-transit photometry was to attempt to measure the stellar rotation period. The parent star is relatively active, with a chromospheric activity index $S=0.525$ (Wright et al. 2004) and $\log R_{H K}^{\prime}=-4.4$, raising the possibility of measuring the rotation period through starspot-induced quasi-periodic flux variations. As we explain, the measurements of both the rotation period and the Rossiter-McLaughlin effect permit the determination of the true angle between the orbit normal and stellar rotation axis (as opposed to the angle between the projections of those vectors on the sky, which was measured by Winn et al. 2006).

This paper is organized as follows. In $\S 2$ we present photometry of eight different transits, along with nightly photometry over two consecutive observing seasons. In particular, $\S 2.2$ presents the out-of-transit photometry and the estimation of the stellar rotation period. In $\S 3$ we describe the parametric model that was fitted to the data, and in $\S 4$ we present the results for the planetary, stellar, and orbital parameters, as well as the new transit ephemerides, a limit on the orbital eccentricity, and the threedimensional spin-orbit alignment. Section 5 is a summary.

\section{OBSERVATIONS AND DATA REDUCTION}

Our observations took place in 2005 and 2006 using telescopes at four different observatories. Each of the sections below presents the photometry from a given observatory. Altogether, we 
TABLE 1

Characteristics of Transit Data

\begin{tabular}{|c|c|c|c|c|c|c|}
\hline 2005 Nov $28 \ldots \ldots \ldots \ldots \ldots \ldots$ & T10 APT $0.8 \mathrm{~m}$ & $(b+y) / 2$ & 1.44 & 0.0045 & 0.00250 & 4.2 \\
\hline 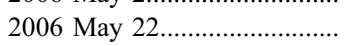 & T10 APT $0.8 \mathrm{~m}$ & $(b+y) / 2$ & 1.44 & 0.0024 & 0.00090 & 3.0 \\
\hline 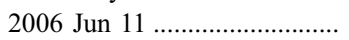 & T10 APT $0.8 \mathrm{~m}$ & $(b+y) / 2$ & 1.30 & 0.0019 & 0.00140 & 5.3 \\
\hline 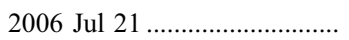 & FLWO $1.2 \mathrm{~m}$ & $z$ & 0.23 & 0.0023 & 0.00035 & 3.3 \\
\hline 2006 Sep 10 & FLWO $1.2 \mathrm{~m}$ & $z$ & 0.23 & 0.0022 & 0.00020 & 2.0 \\
\hline
\end{tabular}

observed eight different transits. Table 1 gives a summary of the characteristics of the data from each transit, and Table 2 gives the final photometry. A telescope at Fairborn Observatory (§ 2.2) was also used to monitor the out-of-transit flux of HD 189733 over the two observing seasons.

\subsection{Fred Lawrence Whipple Observatory}

We observed the transits of UT 2006 July 21 and September 10 with the $1.2 \mathrm{~m}$ telescope at the Fred L. Whipple Observatory (FLWO) on Mount Hopkins, Arizona. ${ }^{10}$ We used KeplerCam, which has one $4096^{2}$ Fairchild 486 back-illuminated CCD, with a $23.1^{\prime} \times 23.1^{\prime}$ field of view. For our observations we used $2 \times 2$ binning, which gives a scale of $0.68^{\prime \prime}$ per binned pixel, a readout and setup time of $11 \mathrm{~s}$, and a typical readout noise of $7 e^{-}$per binned pixel. We observed both transits through the Sloan Digital Sky Survey (SDSS) $z$-band filter in order to minimize the effect of color-dependent atmospheric extinction on the relative photometry and to minimize the effect of limb darkening on the transit light curve. We deliberately defocused the telescope such that the full width at half-maximum (FWHM) of stellar images was about seven binned pixels $\left(4.8^{\prime \prime}\right)$, in order to permit a consistent exposure time of $5 \mathrm{~s}$. We used automatic guiding to keep the image registration as constant as possible. We also obtained dome flat exposures and zero-second (bias) exposures at the beginning and the end of each night.

On UT 2006 July 21, the sky conditions began partly cloudy but gradually improved as the night went on. We observed the target star as it rose from an air mass of 1.19 to 1.01 and then descended to an air mass of 1.23 . There was a 15 minute interruption after second contact, due to clouds. On UT 2006 September 10, the sky conditions were mainly clear (but not all-sky photometric). We followed the target star from an air mass of 1.02 to

${ }^{10}$ The data from the first of these two transits have already been presented by Winn et al. (2006).

TABLE 2

Photometry OF HD 189733

\begin{tabular}{ccccc}
\hline \hline Telescope & Filter & HJD & Relative Flux & Uncertainty \\
\hline FLWO 1.2 m....... & $z$ & $2,453,937.71893$ & 1.0037 & 0.0027 \\
& $z$ & $2,453,937.71909$ & 0.9963 & 0.0027 \\
& $z$ & $2,453,937.71925$ & 1.0007 & 0.0027 \\
\hline
\end{tabular}

Notes.-Table 2 is published in its entirety in the electronic edition of the Astronomical Journal. A portion is shown here for guidance regarding its form and content. The data are also available from the authors upon request. The time stamps represent the Heliocentric Julian Date at the time of midexposure.
2.5 , although we discarded the data taken at air mass $>1.9$ because of their much poorer quality. There was a 15 minute interruption prior to third contact, due to a computer crash, which also caused a change in image registration and focus.

We used standard IRAF ${ }^{11}$ procedures for the overscan correction, trimming, bias subtraction, and flat-field division. We performed aperture photometry of HD 189733 and 14 nearby and necessarily fainter stars. The light curve of each comparison star was normalized to have unit median, and the mean of these normalized light curves was taken to be the comparison signal. The light curve of HD 189733 was divided by the comparison signal and corrected for residual systematic effects by dividing out a linear function of time. The zero point and slope of the linear function were determined as part of the model-fitting procedure, as explained in $\S 4$. Figure 1 shows the final light curves, along with a time-averaged composite light curve created from the two data sets.

\subsection{Fairborn Observatory}

We used the T10 $0.8 \mathrm{~m}$ automated photometric telescope (APT) at Fairborn Observatory, in Arizona, to observe four complete transits of HD 189733b and to monitor the out-of-transit stellar flux. The T10 APT is equipped with a two-channel precision photometer employing two EMI 9124QB bi-alkali photomultiplier tubes to make simultaneous measurements in the Strömgren $b$ and $y$ passbands. The APT measures the difference in brightness between a program star and a nearby constant comparison star (or stars) with a typical precision of 0.0015 mag for bright stars $(V<8.0)$. For the HD 189733 transits, we used the comparison star HD $189410(V=5.68, B-V=0.34, \mathrm{~F} 0)$. The differential magnitudes were reduced with nightly extinction coefficients and transformed to the Strömgren system with yearly mean transformation coefficients. To improve our photometric precision, we combined the separate $b$ and $y$ differential magnitudes into a single $(b+y) / 2$ passband. For additional information on the telescopes, photometers, observing procedures, and data reduction techniques, see Henry (1999) and Eaton et al. (2003).

We observed the transits of UT 2005 November 28, 2006 May 2, 2006 May 22, and 2006 June 11. On each of those nights, the differential magnitude of HD 189733 was recorded for the 35 hours bracketing the expected time of midtransit. The transit light curves are shown in the top four panels of Figure 2. Like the FLWO data, these data have been corrected by a linear function

\footnotetext{
11 The Image Reduction and Analysis Facility (IRAF) is distributed by the National Optical Astronomy Observatory, which is operated by the Association of Universities for Research in Astronomy, Inc., under cooperative agreement with the National Science Foundation.
} 

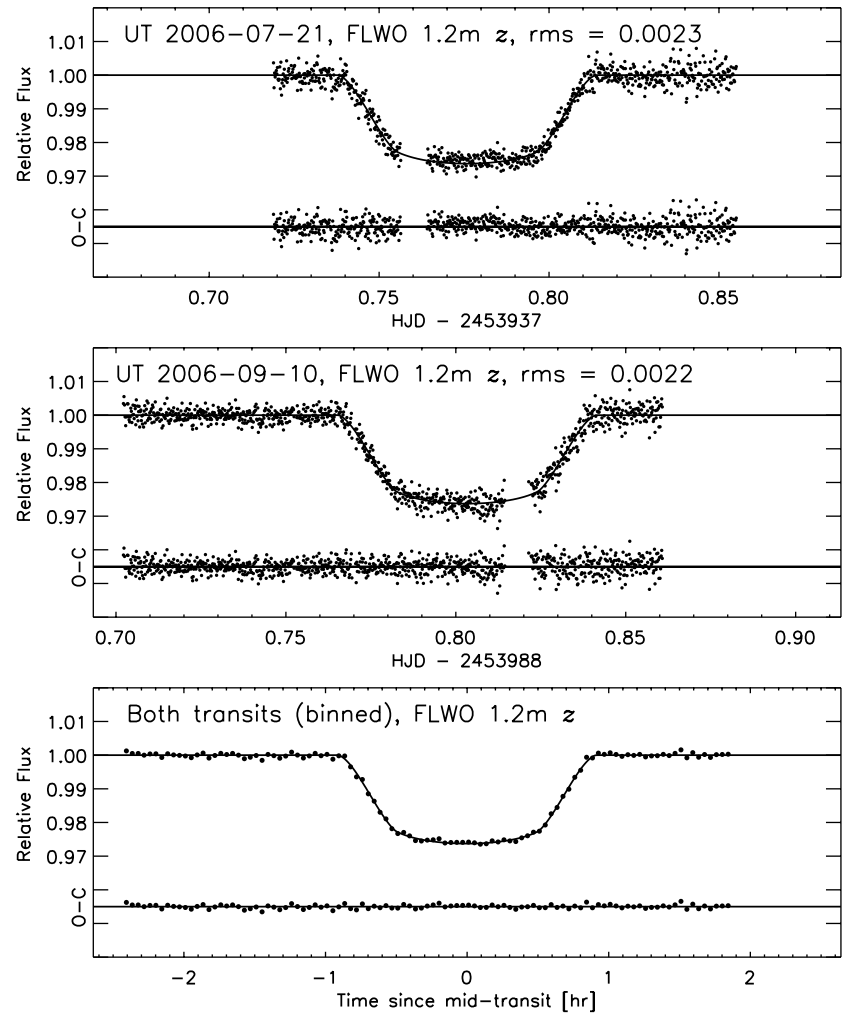

Fig. 1.- Photometry of HD 189733 in the $z$ band, using the FLWO $1.2 \mathrm{~m}$ telescope and KeplerCam. These data were used to estimate the planetary, stellar, and orbital parameters (see $\S 3$ ). The bottom panel is a composite light curve created from both data sets, after time-shifting the earlier transit and averaging into 1 minute bins. The residuals (observed-calculated) are plotted beneath the data.

of time that was determined as part of the fitting procedure (see $\S 4)$.

In addition, we also measured the out-of-transit flux with the APT on 93 different nights spanning two observing seasons between 2005 October and 2006 July. Three comparison stars were observed on each night. The out-of-transit flux measurements are shown in the top two panels of Figure 3. The flux varied erratically during the 2005 observing season and at the beginning of the 2006 season. However, a quasi-periodic signal became evident near the end of our 2006 season observations with a peakto-peak amplitude of $1.3 \%$ and a period of about 13 days, although only 2.5 cycles were observed.

This type of photometric behavior - erratic and occasionally quasi-periodic variation - is common among chromospherically active stars, especially stars with low or intermediate levels of activity (see, e.g., Henry et al. 1995). The photometric variability in these stars arises from photospheric starspots and plages that are carried into and out of view by the stellar rotation. In the case of HD 189733, the spots cover only $\sim 1 \%$ of the stellar surface at any moment, and their distribution on the star changes significantly on the rotational timescale. A periodogram of the quasiperiodic portion of the light curve (indicated by the filled circles in Fig. 3, middle) shows a strong peak at 13.4 days, which we take to be the stellar rotation period. We estimated the uncertainty by recalculating the light curve using each of the three different comparison stars; the standard deviation of the results was 0.4 days. Thus, in what follows, we adopt the value $P_{\text {rot }}=13.4 \pm$ 0.4 days. Since this result is only based on 2.5 cycles, continued observations are warranted to check our estimate. There may be additional errors because of differential rotation and the variations in spot positions and intensities on the rotational timescale.
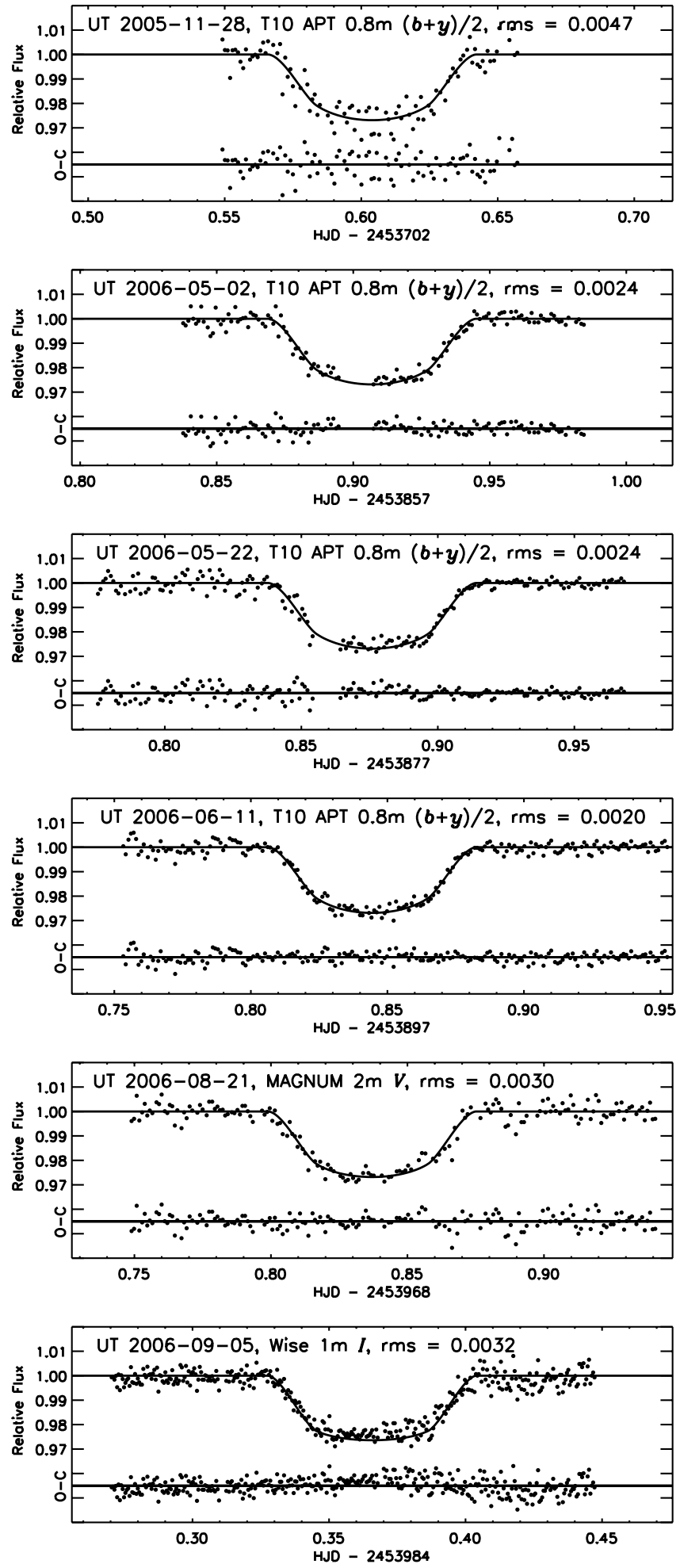

Fig. 2.- Relative photometry of HD 189733 during six different transits. The date, telescope, and filter are identified on each panel. These data were used only for measurements of the times of transit (see $\S 3$ ). The model, shown as the solid line, is based on the model derived from the FLWO $z$-band data after changing the limb-darkening parameters appropriately. In all cases, the residuals (observed-calculated) are plotted beneath the data.

Figure 3 (bottom) shows the flux as a function of rotational phase during the epoch of quasi-periodic variation. A periodogram of the entire data set also shows a peak at 13.4 days, along with a peak at 6.7 days, presumably from a time period when starspots occurred on both sides of the star. 

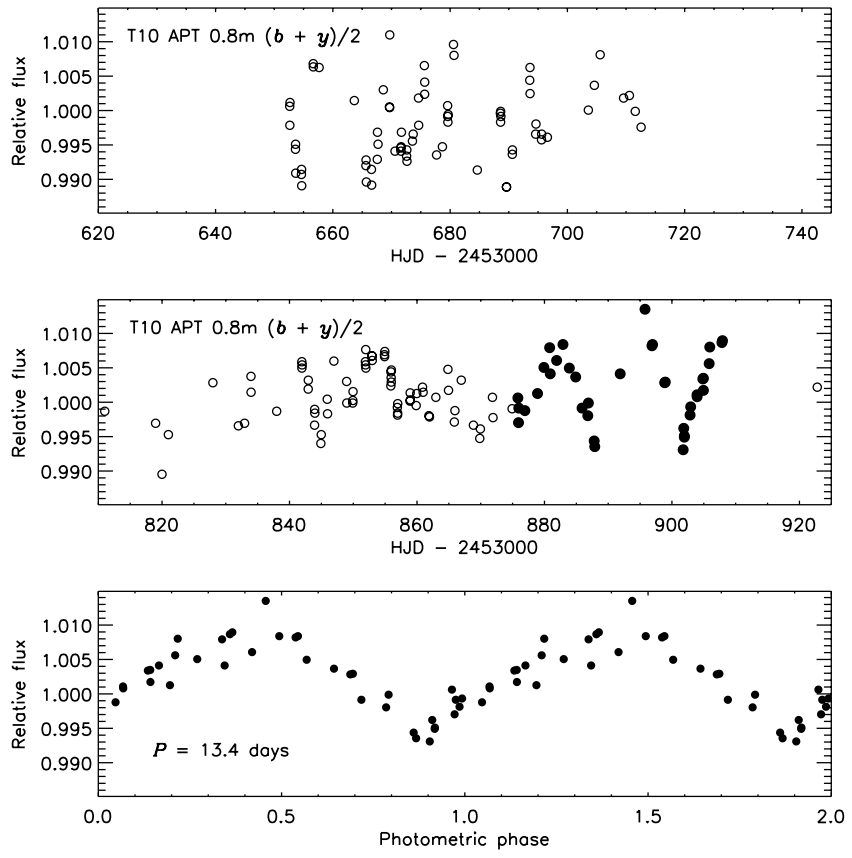

Fig. 3.- Relative photometry of HD 189733 , from the T10 $0.8 \mathrm{~m} \mathrm{APT}$ at Fairborn Observatory. The top two panels show data from the 2005 and 2006 observing seasons, respectively. The filled circles show the portion of data that was used in the periodogram analysis. This subset of the data is plotted in the bottom panel as a function of the photometric phase.

\subsection{MAGNUM Observatory}

We observed the transit of UT 2006 August 21 with the $2 \mathrm{~m}$ telescope at the Multicolor Active Galactic Nuclei Monitoring (MAGNUM) observatory on Haleakala, Hawaii (Kobayashi et al. 1998; Yoshii 2002; Yoshii et al. 2003). This is the same transit that was observed spectroscopically by Winn et al. (2006). We used the multicolor imaging photometer (MIP), which allows for simultaneous observation with an optical $1024^{2}$ SITE CCD and an infrared SBRC InSb $256^{2}$ detector. In this case, we used only the optical detector, because the infrared detector was saturated even in very short exposures. We observed in the $V$ band. Because the MIP field of view is only $1.5^{\prime} \times 1.5^{\prime}$ and there are no good comparison stars within this small field, we nodded repeatedly between the target star and a calibration star, with $1 \mathrm{~s}$ exposures. The calibration star was HD $190449(V=8.12, B-V=0.79$, K0). The average interval between exposures of HD 189733 was $46 \mathrm{~s}$. The median FWHM of stellar images was $1.2^{\prime \prime}$.

We reduced the images with the standard MIP pipeline described by Minezaki et al. (2004). We then performed aperture photometry on HD 189733 and HD 190449, using an aperture radius of $6.65^{\prime \prime}$ and a sky annulus ranging in radius from $6.65^{\prime \prime}$ to $9.42^{\prime \prime}$. To produce a comparison signal, the time series for HD 190449 was boxcar-smoothed (with a width of 4 points, or 3 minutes) and then linearly interpolated onto the time stamps of the HD 189733 data. Then the HD 189733 time series was divided by this comparison signal. A few extreme outlying points were rejected. To remove residual systematic errors in the out-of-transit flux determination, we divided by a linear function of time that was determined as part of the fitting procedure (see $\S 4)$. The final light curve is shown in the fifth panel of Figure 2.

\subsection{Wise Observatory}

We observed the transit of 2006 September 5 with the $1 \mathrm{~m}$ telescope at Wise Observatory, in Israel. We used a Tektronix $1024^{2}$ back-illuminated CCD detector, giving a pixel scale of $0.7^{\prime \prime}$ and a field of view of $11.9^{\prime} \times 11.9^{\prime}$. We observed through a Johnson $I$ filter, the reddest optical band available on this camera. The exposure time was $10 \mathrm{~s}$ and the telescope was defocused in order to avoid saturation. Automatic guiding was used to keep the image registration constant throughout the night. We also obtained sky flat exposures at sunset and zero-second (bias) exposures at the beginning of and during the night. We performed the data reduction and photometry using very similar procedures to those that were used on the FLWO data ( $\$ 2.1)$. Figure 2 (bottom) shows the final light curve.

\section{DETERMINATION OF SYSTEM PARAMETERS}

To estimate the planetary, stellar, and orbital parameters, and the times of transit, we fitted a parameterized model to the transit photometry. The model and the fitting method were similar to those described in previous TLC papers (see, e.g., Winn et al. 2007b), except that in this case we accounted for correlated noise, as described below. The model is based on a Keplerian orbit of a star (with mass $M_{\star}$ and radius $R_{\star}$ ) and a single planet $\left(M_{p}, R_{p}\right)$ about its center of mass. For most of the analysis we assumed that the orbital eccentricity $e$ is zero, because the expected timescale of tidal circularization is short in the absence of excitations from other planets (see, e.g., Rasio et al. 1996; Trilling 2000; Dobbs-Dixon et al. 2004; Adams \& Laughlin 2006). However, in $\S 4.3$ we discuss the empirical upper limits on the orbital eccentricity. The orbit has a period $P$ and an inclination $i$ relative to the sky plane. We define the coordinate system such that $0^{\circ} \leq i \leq 90^{\circ}$. It is often useful to refer to the impact parameter $b \equiv a \cos i / R_{\star}$ (where $a$ is the semimajor axis) rather than the inclination.

Because one of our goals was to measure the individual transit times, we allowed each transit to have an independent value of $T_{c}$, the transit midpoint, rather than forcing them to be separated by exact multiples of the orbital period. Thus, the only effect of $P$ on the model is to determine the semimajor axis $a$ for a given value of the total mass. We fixed $P=2.218575$ days, the value determined by Bouchy et al. (2005) and Hébrard \& Lecavelier Des Etangs (2006) from the detection of transits in the Hipparcos database. The uncertainty of 0.000003 days was negligible for our purposes, although we were able to use the resulting values of $T_{c}$ to produce an independent estimate of the period, as described in $\S 5$.

Neither $M_{\star}$ nor $M_{p}$ can be determined from photometry alone. As we have done in previous TLC analyses, we fixed $M_{\star}$ at a value that is based on an analysis of the stellar spectrum and other observable properties. We then used the scaling relations $R_{p} \propto M_{\star}^{1 / 3}$ and $R_{\star} \propto M_{\star}^{1 / 3}$ to estimate the systematic error associated with the uncertainty in $M_{\star}$. In this case we adopted the value $M_{\star}=$ $0.82 \pm 0.03 M_{\odot}$ (Bouchy et al. 2005). The planetary mass $M_{p}$ hardly affects the photometric model at all, but for completeness we used the value $M_{p}=1.13 M_{\text {Jup }}$ (Winn et al. 2006).

To calculate the relative flux as a function of the projected separation of the planet and the star, we employed the analytic formulas of Mandel \& Agol (2002) to compute the integral of the intensity over the unobscured portion of the stellar disk. We assumed the limb-darkening law to be quadratic,

$$
\frac{I_{\mu}}{I_{1}}=1-u_{1}(1-\mu)-u_{2}(1-\mu)^{2},
$$

where $I$ is the intensity and $\mu$ is the cosine of the angle between the line of sight and the normal to the stellar surface. We fixed the limb-darkening coefficients at the values calculated by Claret $(2000,2004)$ for observations of a star with the observed spectral 
properties. $^{12} \mathrm{We}$ also investigated the effect of fitting for the limb-darkening parameters, as discussed below. In addition, the light curves exhibited gradients in the out-of-transit data, probably due to differential extinction between the target star and the comparison stars, or some other systematic error. For this reason, each of the eight data sets was modeled with two extra parameters: the out-of-transit flux $f_{\text {oot }}$ and a time gradient $\alpha$.

The fitting statistic was

$$
\chi^{2}=\sum_{j=1}^{N_{f}}\left[\frac{f_{j}(\mathrm{obs})-f_{j}(\mathrm{calc})}{\sigma_{j}}\right]^{2},
$$

where $f_{j}(\mathrm{obs})$ is the flux observed at time $j, \sigma_{j}$ controls the relative weights of the data points, and $f_{j}$ (calc) is the calculated value. In order to derive realistic uncertainties on the parameters, it is important for $\sigma_{j}$ to include not only measurement errors but also any unmodeled systematic effects. Of particular importance is the timescale of the systematic effects. Correlated noise effectively reduces the number of independent data points and correspondingly increases the uncertainties in the model parameters, an issue that Pont et al. (2006) and Gillon et al. (2006) have recently raised in the context of transit photometry.

Our approach to this problem was as follows. First, for each of the eight transits, we rescaled the instrumental uncertainties such that $\chi^{2} / N_{\text {dof }}=1$ for the best-fitting model. The resulting uncertainties are those that are given in Table 2. Second, we followed the procedure of Gillon et al. (2006) to decompose the observed noise into "white noise" (that which averages down as $1 / \sqrt{N}$, where $N$ is the number of data points) and "red noise" (that which does not average down over some specified time interval). Specifically, we calculated the standard deviation of the residuals $(\sigma)$ and the standard deviation of the time-averaged residuals $\left(\sigma_{N}\right)$. The averaging time was $1 \mathrm{hr}$ (a timescale comparable to the transit event), corresponding to a number $N$ of data points that depended on the cadence of observations. Then we solved for the white noise $\sigma_{w}$ and red noise $\sigma_{r}$ from the following system of equations:

$$
\begin{aligned}
& \sigma_{1}^{2}=\sigma_{w}^{2}+\sigma_{r}^{2}, \\
& \sigma_{N}^{2}=\frac{\sigma_{w}^{2}}{N}+\sigma_{r}^{2} .
\end{aligned}
$$

Finally, to account approximately for the effective reduction in the number of independent data points, we rescaled the $\sigma_{j}$ in equation (2) by the factor $\sigma_{r} /\left(\sigma_{w} / \sqrt{N}\right)$.

The results for $\sigma_{w}, \sigma_{r}$, and the rescaling factor for each data set are given in Table 1 . These results are not very sensitive to the choice of averaging time. Any choice between 15 minutes and a few hours gave similar results. Among the data sets are wide disparities in the degree of red noise, ranging over a factor of 10 . By far the best data, in the sense of the smallest noise correlations, are from the FLWO $1.2 \mathrm{~m}$ telescope and KeplerCam $(\S 2.1)$. For this reason we decided to estimate the system parameters using only the FLWO data and to use the other data sets only to determine transit times and as a consistency check on the FLWO results. (Had we included the other data sets, their statistical weight would have been much smaller.)

In short, we used the FLWO data to solve for the two bodies' radii $\left(R_{\star}\right.$ and $\left.R_{p}\right)$, the orbital inclination $(i)$, the midtransit time

\footnotetext{
12 Specifically, we used the tabulated values for an ATLAS model with $T_{\text {eff }}=$ $5000 \mathrm{~K}, \log g=4.5(\mathrm{cgs}), \log [\mathrm{M} / \mathrm{H}]=0.0$, and $v_{t}=2.0 \mathrm{~km} \mathrm{~s}^{-1}$. For the $z$ band, $u_{1}=0.32$ and $u_{2}=0.27$
}

\begin{tabular}{|c|c|}
\hline Parameter & Value \\
\hline$\left(R_{\star} / R_{\odot}\right)\left(M_{\star} / 0.82 M_{\odot}\right)^{-1 / 3}$. & $0.753 \pm 0.023$ \\
\hline$\left(R_{p} / R_{\text {Jup }}\right)\left(M_{\star} / 0.82 M_{\odot}\right)^{-1 / 3}$ & $1.156 \pm 0.044$ \\
\hline 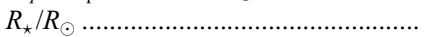 & $0.753 \pm 0.025$ \\
\hline 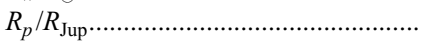 & $1.156 \pm 0.046$ \\
\hline$R_{p} / R_{\star} \ldots$ & $0.1575 \pm 0.0017$ \\
\hline$\left(R_{p} / a\right)^{2} \ldots .$. & $0.000313 \pm 0.000025$ \\
\hline$R_{\star} / a \ldots \ldots \ldots \ldots$ & $0.1124 \pm 0.0034$ \\
\hline$i(\operatorname{deg}) \ldots \ldots \ldots \ldots \ldots \ldots \ldots$ & $85.76 \pm 0.29$ \\
\hline 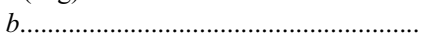 & $0.658 \pm 0.027$ \\
\hline 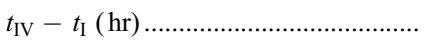 & $1.827 \pm 0.029$ \\
\hline$t_{\mathrm{II}}-t_{\mathrm{I}}($ minutes $) \ldots \ldots . .$. & $24.6 \pm 1.9$ \\
\hline
\end{tabular}

TABLE 3

System Parameters of HD 189733

$\left(T_{c}\right)$, the out-of-transit flux $\left(f_{\text {oot }}\right)$, and a time gradient $(\alpha)$ for each of the two transits. We then fixed $R_{\star}, R_{p}$, and $i$ at the bestfitting values, and fitted each of the remaining six data sets to find $T_{c}, f_{\text {oot }}$, and $\alpha$.

To solve for the model parameters and their uncertainties, we used a Markov Chain Monte Carlo (MCMC) algorithm (see, e.g., Tegmark et al. 2004). Our jump function was the addition of a Gaussian random number to each parameter value. We set the perturbation sizes such that $\sim 20 \%$ of jumps are executed. We created 10 independent chains, each with 500,000 points, starting from random initial positions, and discarded the first $20 \%$ of the points in each chain. The Gelman \& Rubin (1992) $R$-statistic was within $0.2 \%$ of unity for each parameter, a sign of good mixing and convergence. We merged the chains and took the median value of each parameter to be our best estimate, and the standard deviation as the $1 \sigma$ uncertainty.

\section{RESULTS}

The results are given in Table 3. Along with the results for the model parameters, we have provided results for some useful derived quantities such as the impact parameter $b$, the radius ratio $\left(R_{p} / R_{\star}\right)$, and the fraction $\left(R_{p} / a\right)^{2}$ (which gives the fraction of starlight reflected by the planet, for an albedo of unity). We also report the calculated values of the full transit duration (the time between first and fourth contact, $t_{\mathrm{IV}}-t_{\mathrm{I}}$ ), and the partial transit duration (the time between first and second contact, or between third and fourth contact). ${ }^{13}$

\subsection{Stellar and Planetary Radii}

The result for the stellar radius is $R_{\star}=0.753 \pm 0.025 R_{\odot}$. The uncertainty is dominated by the statistical error of $0.023 R_{\odot}$ (3.1\%). The covariance with the uncertainty in the stellar mass produces an additional error of $0.009 R_{\odot}(1.2 \%)$, which we have added in quadrature to the statistical error to arrive at the net uncertainty of $0.025 R_{\odot}$. We find the planetary radius to be $R_{p}=$ $1.156 \pm 0.046 R_{\text {Jup }}$, where the uncertainty is again dominated by the statistical error of $0.044 R_{\mathrm{Jup}}$.

To test the robustness of these results, we performed some additional fits. We gauged the importance of the choice of limbdarkening law by refitting the data under different assumptions. When we allowed the limb-darkening coefficients $u_{1}$ and $u_{2}$ to be free parameters rather than holding them fixed, we found $R_{\star}=$ $0.755 R_{\odot}$ and $R_{p}=1.163 R_{\text {Jup }}$, well within the $1 \sigma$ error of our

\footnotetext{
13 Although the partial transit duration is listed as $t_{\mathrm{II}}-t_{\mathrm{I}}$ in Table 3, all of the results in Table 1 are based on the entire light curves, including both ingress and egress data. Our model assumes $t_{\mathrm{II}}-t_{\mathrm{I}}=t_{\mathrm{IV}}-t_{\mathrm{III}}$.
} 
original analysis. (The optimized limb-darkening coefficients for the $z$ band were $u_{1}=0.35$ and $u_{2}=0.22$, as compared to the theoretical values of $u_{1}=0.32$ and $u_{2}=0.27$.) Likewise, the results changed by only $0.25 \sigma$ when we used a linear limb-darkening law $\left(u_{2}=0\right)$, regardless of whether the linear limb-darkening coefficient was fixed or taken to be a free parameter. As another check, we fitted each of the eight transit data sets separately, solving for the parameters $\left\{R_{\star}, R_{p}, i, T_{c}, \alpha, f_{\text {oot }}\right\}$ in each case. Although the statistical power of the FLWO photometry was the greatest, as noted previously, all eight sets of results agreed within their calculated error bars. In particular, the unweighted "ensemble averages" of the results from the six non-FLWO data sets were $R_{\star}=0.760 R_{\odot}$ and $R_{p}=1.148 R_{\text {Jup }}$, again in agreement with our original analysis.

How do these results compare to the previous analyses of HD 189733 by Bouchy et al. (2005) and Bakos et al. (2007)? An important difference is that those authors decided not to determine $R_{\star}$ from the transit photometry. ${ }^{14}$ Rather, those authors used estimates of $R_{\star}$ based on an analysis of other observable properties of the star. Bouchy et al. (2005) used measurements of the star's parallax, effective temperature, surface gravity, and metallicity, in comparison with the outputs of stellar evolution models, and concluded that $R_{\star}=0.76 \pm 0.01 R_{\odot}$. Likewise, Bakos et al. (2007) investigated four different ways of determining the stellar radius, based on broadband colors, spectral properties, and model isochrones, and found a stellar radius in the range $0.74-0.79 R_{\odot}$. Ultimately, Bakos et al. (2007) adopted the value $0.758 \pm 0.016 R_{\odot}$ based on a calibration of 2 MASS photometry by Masana et al. (2006). Our determination of $R_{\star}$ based on the transit light curve is in agreement with those independent determinations; the mutual agreement constitutes an important consistency check on the data and our analysis.

Our value for the planetary radius agrees with the value $R_{p}=$ $1.154 \pm 0.033 R_{\text {Jup }}$ found by Bakos et al. (2007). While the Bakos et al. (2007) result would appear to be more precise, the comparison is somewhat misleading because Bakos et al. (2007) were not simultaneously fitting for $R_{\star}$, as noted above. If we follow their procedure of fixing $R_{\star}=0.758 \pm 0.016 R_{\odot}$, then our result for the planetary radius becomes more precise: $R_{p}=$ $1.164 \pm 0.028 R_{\text {Jup }}$. Both our result and that of Bakos et al. (2007) disagree with that of Bouchy et al. (2005), who found $1.26 \pm 0.03 R_{\text {Jup }}$ based on a $B$-band light curve from the $1.2 \mathrm{~m}$ telescope at the Observatoire de Haute-Provence (OHP). This discrepancy was traced by Bakos et al. (2007) to systematic errors in the OHP photometry. When the $B$-band light curve was recalculated using a greater number of comparison stars, the transit depth decreased by $20 \%$ and the inferred planetary radius shrank accordingly.

How does the planetary radius compare to theoretical expectations, given its mass $\left(M_{p}=1.13 \pm 0.03 M_{\text {Jup }}\right.$; Winn et al. 2006) and its proximity to its parent $\operatorname{star}(a=0.031 \mathrm{AU})$ ? Fortney et al. (2007) have recently provided a wide range of theoretical predictions for exoplanet radii. Their calculations are for a solarmass star, but at their suggestion we can rescale the semimajor axis to compensate for the lower luminosity of HD 189733. Assuming $\left(L_{\star} / L_{\odot}\right)=\left(M_{\star} / M_{\odot}\right)^{3.5}$, if HD 189733 were orbiting the Sun at $a=0.05$ it would receive roughly the same incident flux as it does in its actual orbit. The resulting prediction from Figure 6 of Fortney et al. (2007) is a planetary radius between $\approx 1.05$ and

\footnotetext{
14 While Bakos et al. (2007) did fit for the stellar radius, finding $R_{\star}=$ $0.68 \pm 0.02 R_{\odot}$, they did not trust the result. They quite reasonably suspected that the true error bar was significantly larger than their calculations indicated because of correlated noise in the data.
}

TABLE 4

Midtransit Times of HD $189733 b$

\begin{tabular}{|c|c|}
\hline Epoch $(E)$ & $\begin{array}{c}\text { Midtransit Time } \\
\text { (HJD) }\end{array}$ \\
\hline$-129 \ldots$ & $2,453,702.60416 \pm 0.0032$ \\
\hline . n & $2,453,857.90694 \pm 0.00086$ \\
\hline (..................... & $2,453,877.87598 \pm 0.00094$ \\
\hline 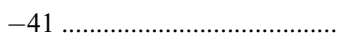 & $2,453,897.84444 \pm 0.0014$ \\
\hline ................. & $2,453,937.77590 \pm 0.00044$ \\
\hline ................. & $2,453,968.83715 \pm 0.0013$ \\
\hline$-2 \ldots \ldots$. & $2,453,984.36592 \pm 0.0013$ \\
\hline . & $2,453,988.80331 \pm 0.00027$ \\
\hline
\end{tabular}

$1.12 R_{\text {Jup }}$, assuming an age of $\sim 4.5 \mathrm{Gyr}$ and depending on whether or not the planet has a massive $\left(25 M_{\oplus}\right)$ core. The corefree prediction for the radius is larger and in agreement with the observed value. However, it seems premature to claim that a massive core is disfavored, given the uncertainties that enter into the calculations and the uncertainty in the age of the system. It does seem safe to say that the radius of HD 189733 does not present a severe theoretical problem, unlike the cases of the apparently "bloated" planets HD 209458b, HAT-P-1b, and WASP-1b (for recent results on those systems, see Knutson et al. 2007; Bakos et al. 2006; Collier Cameron et al. 2007; Charbonneau et al. 2007b).

The mean density of HD $189733 \mathrm{~b}$ is $\rho_{p}=0.91 \pm 0.06 \mathrm{~g} \mathrm{~cm}^{-3}$, which is between the densities of Saturn $\left(0.6 \mathrm{~g} \mathrm{~cm}^{-3}\right)$ and Jupiter $\left(1.2 \mathrm{~g} \mathrm{~cm}^{-3}\right)$. The surface gravity of HD $189733 \mathrm{~b}$ is $g=21 \pm$ $1 \mathrm{~m} \mathrm{~s}^{-2}$, which is also intermediate between Saturn $\left(10 \mathrm{~m} \mathrm{~s}^{-2}\right)$ and Jupiter $\left(25 \mathrm{~m} \mathrm{~s}^{-2}\right)$. We note that whenever $M_{p}$ is measured via the spectroscopic orbit of the star, and $R_{p}$ is measured via transit photometry (as is the case here), then the derived value of $g$ is immune to systematic errors in the parameters of the parent star. This is because the fitting degeneracies are $M_{p} \propto M_{\star}^{2 / 3}$ and $R_{p} \propto M_{\star}^{1 / 3}$, and hence $g \equiv G M_{p} / R_{p}^{2}$ is independent of $M_{\star}^{\star}$. Here this fact is of limited interest, because the error in $R_{p}$ is dominated by statistical error, but it may be of importance in future transit studies. ${ }^{15}$

\subsection{Determination of the Transit Ephemerides}

Table 4 gives the eight transit times measured from our data. We have used these times, along with transit times previously measured by Bakos et al. (2006), to calculate a photometric ephemeris for this system. Although Bakos et al. (2006) reported 15 measured times, we used only four of those data points in our analysis. We did not include the 10 times that were based on only partial observations of the transit. Full transits are greatly preferable, in order to correct (or at least assess) systematic errors using the pre-ingress and post-egress data. In addition, we did not include the $T_{c}$ measurement based on the OHP $B$-band light curve of Bouchy et al. (2005) because of the systematic errors noted previously. What remained were four data points representing four independent measurements of the same event. In combination with our eight data points, we fitted a linear function of transit epoch $E$,

$$
T_{c}(E)=T_{c}(0)+E P,
$$

finding $T_{c}(0)=2,453,988.80336(23) \quad(\mathrm{HJD})$ and $P=$ 2.2185733(19) days, where the numbers in parentheses indicate

\footnotetext{
${ }^{15}$ We thank S. Gaudi for helping us to appreciate this point.
} 


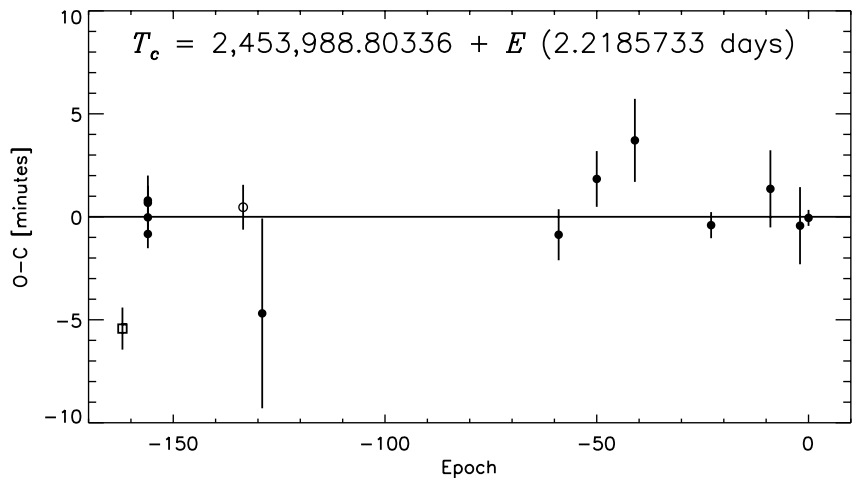

FIG. 4. - Transit and secondary-eclipse timing residuals for HD 189733. The calculated times, using the ephemeris of eq. (5), have been subtracted from the observed times. The filled circles represent data points used in the fit. The square is based on $B$-band data by Bouchy et al. (2005), as reanalyzed by Bakos et al. (2007). The open circle is the secondary-eclipse time measured by Deming et al. (2006).

the $1 \sigma$ uncertainty in the final two digits. The fit had $\chi^{2} / N_{\text {dof }}=$ 1.08 and $N_{\text {dof }}=11$. We chose $E=0$ to correspond to the most precisely known transit time. Our derived period agrees almost exactly with the value of 2.2185730(20) days determined by Bakos et al. (2007), and it is also in agreement with the Hipparcos-based values of 2.2185750(30) days (Bouchy et al. 2005) and $2.218574_{-0.000010}^{+0.00000}$ days (Hébrard \& Lecavelier des Estangs 2006).

Figure 4 is the $O-C$ (observed minus calculated) diagram for the transit times, according to this new ephemeris. The filled circles represent data points used in the fit. There is not yet any pattern in the residuals that would indicate the effect of a perturbing body in the system. The square shows the OHP $B$-band measurement, which is indeed an outlier. The open circle is explained in $\S 4.3$.

\subsection{Limits on the Orbital Eccentricity}

As mentioned previously, one would expect the orbit of a hot Jupiter such as HD 189733 b to be very nearly circular, due to tidal effects. Previous results and our results have all shown that a circular orbit does indeed provide a satisfactory description of the available data. However, it is still interesting to make an empirical determination of the eccentricity, both in the spirit of "what one can measure, one should measure" and also because any additional bodies in the system could excite the orbital eccentricity. We used a two-step procedure to determine the orbital eccentricity.

First, we used our revised ephemeris to interpret the secondaryeclipse timing of Deming et al. (2006). For a nonzero (but small) orbital eccentricity, the time difference between the midpoint of secondary eclipse, $T_{\mathrm{sec}}$, and the time of transit, $T_{\text {tra }}$, may differ from half of the orbital period:

$$
T_{\mathrm{sec}}-T_{\mathrm{tra}} \approx \frac{P}{2}\left(1+\frac{4}{\pi} e \cos \omega\right),
$$

where $\omega$ is the argument of pericenter. Deming et al. $(2006)$ measured the midpoint of a secondary eclipse at HJD 2,453,692.62416 \pm 0.00067 , which is consistent with the $e=0$ prediction of our ephemeris. The open circle in Figure 4 represents the secondaryeclipse measurement. The timing offset of equation (6) is $0.46 \pm$ 1.1 minutes, corresponding to $e \cos \omega=0.00023 \pm 0.00054$.

Second, to determine the other component, $e \sin \omega$, of the eccentricity vector, we used the radial velocities presented by Winn et al. (2006). We used only those 60 velocities measured outside of transits (i.e., not affected by the Rossiter-McLaughlin effect). We performed an MCMC analysis to solve for the Keplerian orbital parameters, as well as a possible long-term velocity gradient, using the same treatment of the measurement errors that Winn et al. (2006) applied to the entire data set. We allowed both $e$ and $\omega$ to be free parameters, but with an a priori constraint on $e \cos \omega$ to enforce compliance with the secondary-eclipse measurement. The result for $e \sin \omega$ was $-0.007 \pm 0.011$.

Hence, both components of the eccentricity vector are consistent with zero, and $e \cos \omega$ is about 20 times more tightly bounded than $e \sin \omega$. Values of $e$ as large as $\approx 0.02$ are allowed, but only for $\omega$ very close to $\pm 90^{\circ}$.

\subsection{Three-Dimensional Spin-Orbit Alignment}

Thanks to the APT data ( $\S 2.2$ ), HD 189733 is the first star with a measured rotation period that also has a transiting planet. Together with the transit photometry and the observation of the Rossiter-McLaughlin effect, this allows for the determination of the angle between the stellar rotation axis and the planetary orbit normal, as anticipated by Queloz et al. (2000). This angle is worth measuring because any significant misalignment may be an indication of perturbative effects during planetary migration, among other reasons (as explained in more detail by Ohta et al. [2005], Winn et al. [2005], and Gaudi \& Winn [2007]).

The true (three-dimensional) angle $\psi$ between the stellar spin axis and the orbital axis is given by the formula

$$
\cos \psi=\cos i_{\star} \cos i+\sin i_{\star} \sin i \cos \lambda,
$$

where $i$ is the orbital inclination, $i_{\star}$ is the inclination of the stellar rotation axis, and $\lambda$ is the angle between the sky projections of the two axes. (For a diagram of the coordinate system, see Fig. 3 of Ohta et al. 2005.) The transit photometry determines $i$ with excellent accuracy. Observations of the Rossiter-McLaughlin effect have been used to determine $\lambda$, but they cannot be used to determine $i_{\star}$ independently. Rather, they are sensitive to $v \sin i_{\star}$, the projected rotation rate of the star. Given $v \sin i_{\star}$ from the Rossiter-McLaughlin observations, ${ }^{16}$ along with $i$ and $R_{\star}$ from the transit photometry, and the stellar rotation period $P_{\text {rot }}$, one can determine $\sin i_{\star}$ via the formula

$$
\sin i_{\star}=v \sin i_{\star}\left(\frac{P_{\text {rot }}}{2 \pi R_{\star}}\right) .
$$

Hence, all of the angles in equation (7) are known. Using the values $\lambda=-1.4^{\circ} \pm 1.1^{\circ}$ and $v \sin i_{\star}=2.97 \pm 0.22 \mathrm{~km} \mathrm{~s}^{-1}$ from Winn et al. (2006), we found $\sin i_{\star}=1.04 \pm 0.09$. By rejecting values of $\sin i_{\star}>1$ as unphysical, and propagating the errors through equation (7), we determined an upper bound on the (mis)alignment angle $\psi$ of $27^{\circ}$ with $95 \%$ confidence. Essentially the same result can be obtained from the approximation $\cos \psi \approx \sin i_{\star}$, which is valid because $i \approx 90^{\circ}$ and $\lambda \approx 0^{\circ}$ are tightly constrained.

This is the first exoplanetary system for which it has been possible to measure $\psi$. The result is consistent with zero, but it is not as precise as the result for the projected angle $\lambda$. How could the measurement of $\psi$ be improved? We have already mentioned some caveats relating to the measurement of the rotation period ( $\S 2.2),{ }^{17}$ but the current uncertainty in $\psi$ is dominated by the error in $v \sin i_{\star}$, which is itself dominated by systematic errors

\footnotetext{
${ }^{16}$ Of course, it is also possible to use a more traditional measurement of $v \sin i_{\star}$, from an analysis of the width of photospheric absorption lines.

17 We note that if the logic in this section is reversed, and one is willing to assume perfect spin-orbit alignment, then the calculated rotation period is $P_{\text {rot }}=12.8 \pm 1.0$ days.
} 
arising from the interpretation of the transit spectra (see Winn et al. 2006). Specifically, the systematic error arose from the "calibration" of the Rossiter-McLaughlin effect using simulated spectra, which was needed because the transit spectra were observed through an $\mathrm{I}_{2}$ cell and analyzed with an algorithm that is nominally designed for measuring Doppler shifts rather than spectral distortions. Further improvement might be achieved through a more sophisticated set of simulations or perhaps by reobserving the Rossiter-McLaughlin effect without the $\mathrm{I}_{2}$ cell (i.e., using a different technique to account for instrumental variations).

\section{SUMMARY}

We have presented photometry of eight complete transits of the exoplanet HD 189733b and modeled the light curves in order to determine the radii of the star and the planet. Our results are consistent with previous results and with theoretical expectations for close-in Jovian planets. Stringent limits on the orbital ec- centricity follow from the measured transit times, in conjunction with a previous detection of the secondary eclipse and with the spectroscopic orbit. We have also presented nightly out-of-transit photometry spanning $2 \mathrm{yr}$, which has revealed the stellar rotation period. We have used this information, along with a previous analysis of the spectroscopic transit, to place an upper bound on the true angle between the stellar rotation axis and the orbital axis. With these developments, HD 189733b has become one of the most thoroughly characterized planets outside of the solar system.

We thank F. Pont and M. Gillon for helpful discussions about correlated noise. We are grateful to G. Marcy, P. Butler, S. Vogt, and E. Turner for their help with the Doppler analysis and for encouragement. A. R. thanks the MIT Undergraduate Research Opportunities Program office for research funding.
Adams, F. C., \& Laughlin, G. 2006, ApJ, 649, 1004

Agol, E., Steffen, J., Sari, R., \& Clarkson, W. 2005, MNRAS, 359, 567

Bakos, G. Á., et al. 2006, ApJ, 650, 1160 2007, ApJ, 656, 552

Bouchy, F., et al. 2005, A\&A, 444, L15

Butler, R. P., Marcy, G. W., Vogt, S. S., \& Apps, K. 1998, PASP, 110, 1389

Charbonneau, D., Brown, T. M., Burrows, A., \& Laughlin, G. 2007a, in Protostars \& Planets V, ed. B. Reipurth, D. Jewitt, \& K. Keil (Tucson: Univ. Arizona Press), 701

Charbonneau, D., et al. 2007b, ApJ, 659, in press

Claret, A. 2000, A\&A, 363, 1081

$$
\text { 2004, A\&A, 428, } 1001
$$

Collier Cameron, A., et al. 2007, MNRAS, 375, 951

Deming, D., Harrington, J., Seager, S., \& Richardson, L. J. 2006, ApJ, 644, 560

Dobbs-Dixon, I., Lin, D. N. C., \& Mardling, R. A. 2004, ApJ, 610, 464

Eaton, J. A., Henry, G. W., \& Fekel, F. C. 2003, in The Future of Small Telescopes in the New Millennium, Vol. 2, ed. T. D. Oswalt (Dordrecht: Kluwer), 189

Fortney, J. J., Marley, M. S., \& Barnes, J. W. 2007, ApJ, in press

Gaudi, B. S., \& Winn, J. N. 2007, ApJ, 655, 550

Gelman, A., \& Rubin, D. B. 1992, Stat. Sci., 7, 457

Gillon, M., Pont, F., Moutou, C., Bouchy, F., Courbin, F., Sohy, S., \& Magain, P. 2006, A\&A, 459, 249

Hébrard, G., \& Lecavelier Des Etangs, A. 2006, A\&A, 445, 341

Henry, G. W. 1999, PASP, 111, 845

Henry, G. W., Fekel, F. C., \& Hall, D. S. 1995, AJ, 110, 2926

\section{REFERENCES}

Holman, M. J., \& Murray, N. W. 2005, Science, 307, 1288

Holman, M. J., et al. 2006, ApJ, 652, 1715

. 2007, ApJ, 655, 1103

Knutson, H., et al. 2007, ApJ, 655, 564

Kobayashi, Y., et al. 1998, Proc. SPIE, 3352, 120

Mandel, K., \& Agol, E. 2002, ApJ, 580, L171

Masana, E., Jordi, C., \& Ribas, I. 2006, A\&A, 450, 735

Minezaki, T., Yoshii, Y., Kobayashi, Y., Enya, K., Suganuma, M., Tomita, H., Aoki, T., \& Peterson, B. A. 2004, ApJ, 600, L35

Miralda-Escudé, J. 2002, ApJ, 564, 1019

Ohta, Y., Taruya, A., \& Suto, Y. 2005, ApJ, 622, 1118

Pont, F., Zucker, S., \& Queloz, D. 2006, MNRAS, 373, 231

Queloz, D., Eggenberger, A., Mayor, M., Perrier, C., Beuzit, J. L., Naef, D., Sivan, J. P., \& Udry, S. 2000, A\&A, 359, L13

Rasio, F. A., Tout, C. A., Lubow, S. H., \& Livio, M. 1996, ApJ, 470, 1187

Tegmark, M., et al. 2004, Phys. Rev. D, 69, 103501

Trilling, D. E. 2000, ApJ, 537, L61

Winn, J. N., Holman, M. J., \& Roussanova, A. 2007a, ApJ, 657, 1098

Winn, J. N., et al. 2005, ApJ, 631, 1215

2006, ApJ, 653, L69

$2007 \mathrm{~b}, \mathrm{AJ}, 133,11$

Wright, J. T., Marcy, G. W., Butler, R. P., \& Vogt, S. S. 2004, ApJS, 152, 261

Yoshii, Y. 2002, in New Trends in Theoretical and Observational Cosmology, ed. K. Sato \& T. Shiromizu (Tokyo: Universal Academy Press), 235

Yoshii, Y., Kobayashi, Y., \& Minezaki, T. 2003, BAAS, 35, 752 